Article

\title{
Culturally and linguistically diverse populations in medical research: perceptions and experiences of older Italians, their families, ethics administrators and researchers
}

\author{
Robyn Woodward-Kron, ${ }^{1}$ Jo-anne Hughson, ${ }^{1}$ Anna Parker, ${ }^{1}$ Agnese Bresin, ${ }^{1}$ John Hajek, ${ }^{1}$ \\ Ute Knoch, ${ }^{1}$ Tuong Dien Phan ${ }^{1,2}$ David Story ${ }^{1}$ \\ ${ }^{1}$ University of Melbourne; ${ }^{2}$ St. Vincent's Hospital, Melbourne, Australia
}

Significance for public health

Many people from culturally and linguistically diverse (CALD) backgrounds remain excluded from medical research such as clinical trials due to a range of language and cultural factors that can be amplified when this population is ageing. This exclusion has implications for the ability of CALD populations to benefit from participating in medical research and for applying research findings to CALD populations. It is essential to develop and implement strategies to include CALD communities in medical research and to uphold the ethical obligation of obtaining informed consent to research. The findings of this study have guided the development of a tablet-based resource which can be used in clinical and community contexts to raise awareness about the purpose of medical research. The resource has been carefully designed to be appropriate for participants' cultural background as well as their preferred language and literacy level. Such a resource has potential to address some of the cultural and linguistic barriers to clinical trial participation of CALD populations.

\section{Abstract}

Background. Low-participation of culturally and linguistically diverse (CALD) patients in medical research remains a problem in migrant and refugee destination countries such as Australia. The aims of this study were to explore i) CALD persons' perceptions and experiences of the medical system and medical research, in this case, older Italian Australians; and ii) the views of research professionals on CALD patient participation in medical research.

Design and Methods. A qualitative study was conducted in Melbourne, Australia, in 2015 utilising in-depth interviews and focus groups with four stakeholder groups: older Italian Australians ( $n=21)$; adult children of older Italian Australians ( $\mathrm{n}=10)$; hospital Human Research Ethics Committee administrators $(n=4)$; and clinical researchers $(n=4)$. The data were analysed for content and thematic analysis.

Results. Themes for the CALD and family group were getting by in medical interactions; receptivity to medical research: testing the waters; and, receptivity to technology for support: passive versus active. Themes for the researcher and HREC groups about CALD patient participation in research were: exclusion; cultural factors; and e-consent.

Conclusions. Our findings from four stakeholder perspectives and experiences confirm that there were considerable cultural, linguistic, and resourcing barriers hindering the participation of older Italian-Australians in medical research. Furthermore, our findings showed that in this study setting there were few enabling strategies in place to address these barriers despite the national ethics guidelines for equitable participation in research. The findings informed the creation of a multimedia tool whose purpose is to address and improve representation of CALD groups in clinical research.

\section{Introduction}

Culturally and linguistically diverse (CALD) groups in Australia, as in other migrant destination countries, suffer from poorer health than the general population, ${ }^{1}$ yet their use of health services tends to be lower. ${ }^{2}$ Levels of health literacy amongst CALD groups are also low. ${ }^{3}$ Research has confirmed that equitable access for CALD groups in the health system will only improve when cultural needs and sensitivities are taken into account, ${ }^{4,5}$ or when CALD groups are provided with culturally safe services. One key area where CALD groups currently miss out on health care opportunities is in clinical trials. ${ }^{6}$ Patients can benefit from participating in a trial, even if they are receiving a placebo or usual care, because they receive closer monitoring, and are taking a more active role in their health care. ${ }^{7}$

Over $50 \%$ of people in some Australian urban centres have a nonEnglish speaking background. ${ }^{8}$ Currently, many of these people are overlooked in clinical trials because of real and/or perceived issues about their level of understanding and consequent ability to provide informed consent. Excluding large sections of the population from research has significant ramifications: trial data are not generalisable to the target populations, ${ }^{9}$ and trial participants may not be representative of those who can most benefit from new therapies or interventions. ${ }^{6}$ The Australian National Statement on Ethical Conduct in Human Research identifies the need to accommodate cultural factors in research participation and to present information to participants in ways that support them and help them to make informed choices. ${ }^{10}$ These ways should take into account the best modalities for information communication; the need for accurate and reliable translations; cultural effects on language comprehension; educational background and level; age; and visual, hearing or communication impairment.

Current information provided to possible participants in clinical research is usually in the form of highly complex written documents in English. ${ }^{11,12}$ Participation requires patients to provide their informed consent, acknowledging they have understood the ethical dimensions of their involvement. The cultural background of CALD populations may not provide them with knowledge of concepts such as medical research, clinical trial, and ethics. ${ }^{13,14}$ Indeed, these concepts can be poorly understood amongst the English-speaking background Australian population. ${ }^{15}$ In addition, people from CALD backgrounds may have different attitudes and beliefs about health to those from an Anglo-Celtic, predominantly English-speaking background. ${ }^{16}$ Traditional informed consent processes tend not to accommodate cultural differences such as a reluctance to sign documents. ${ }^{17}$ Thus, it is important to conduct research on group beliefs about health, expectations, knowledge and health system experiences of CALD groups to gain insights into these differences and how to appropriately address them. ${ }^{18}$ 
This study focussed on older Italian Australians, a CALD group identified by Australian metropolitan clinicians as constituting a significant proportion of local patient populations, but excluded from clinical trials because of language and cultural issues. The aims of this study were to explore: i) perceptions and experiences of the medical system and medical research by CALD patients; and ii) the views of research professionals on CALD patient participation in medical research. A third aim was for these findings to inform the development of a multimedia resource for medical research and clinical trials. This included exploring participant perceptions of the role of technology to facilitate recruitment and consenting of CALD patients, in this case older Italian Australians. Multilingual multimedia technology development for application in research recruitment is a new research area with no documented findings as to the efficacy of such resources in CALD populations (to our knowledge). However, multimedia technologies are increasingly being used in other populations with some promising results, ${ }^{19-21}$ and they present novel opportunities for overcoming known barriers to inclusion of CALD groups in medical research around language, culture and resources issues.

\section{Design and Methods}

To obtain an in-depth understanding of the preferences and experiences of this group, a qualitative research approach was adopted, conducting interviews and focus groups with four key stakeholder groups: older people in the Italian community (CALD group), family members (Family group), clinical researchers (Researcher group), and hospital human research ethics administrators (HREC Group).

A priority in this study was to respect and accommodate the cultural and linguistic profile of the target population in accordance with the National Statement on Ethical Conduct in Human Research. ${ }^{10}$ The study's design and conduct was informed by the literature on the involvement of CALD groups in medical research. ${ }^{22-24}$ For the initial phase of the project, our approach incorporated several key strategies used to overcome potential trust, communication, cultural sensitivity, and access/mobility facilitation issues. To maximise trust, a community-based approach was taken whereby the CALD participants were recruited from the social network of the interviewer, and interviews took place in a familiar, convenient and safe environment to the participants (in their homes or in a community centre). ${ }^{25}$ To maximise ease of communication and/or limit potential embarrassment due to low literacy or low English proficiency, in-person interviews were conducted rather than written questionnaires and in the preferred/dominant language of the participants. The interview questionnaire for the CALD group was translated into Italian by one team member, who is an Italian language professor (JHa), and reviewed for linguistic and cultural appropriateness by the native Italian team member (AB).

\section{Study context and setting}

The study was conducted in Melbourne, Australia, a highly culturally and linguistically diverse city of both established post Second World War and new migrant groups. Italians constitute the third largest non-English-speaking background migrant group in Greater Melbourne and are clustered in the upper age groups; ${ }^{26}$ the largest influx of Italian migrants arrived in Australia in the 1950s and 1960s and migration throughout this period accounts for $78 \%$ of the total Italian migration to Melbourne. ${ }^{27}$ This group is characterised by relatively low levels of education, low literacy levels, low English proficiency and high levels of Italian/dialect language retention. ${ }^{28}$

An inter-disciplinary research team was assembled to address the intersection of cultural, linguistic and clinical research factors at play.
The team included researchers from medicine and humanities backgrounds with expertise in clinical trials (DS, TP, AP), Italian language (JHa, AB), cross-cultural and healthcare communication (RWK), language testing (JHa, JHu) and research ethics and governance (AP, DS).

Interviews with older Italians (referred to as the CALD Group) and family members (referred to as the Family group) were conducted in the homes of participants. Two focus groups were also conducted with the CALD group in a community setting - the local Italian senior citizens' centre. The interviews and focus groups with the CALD participants were conducted in Italian by AB. Family member interviews were conducted in English, the preferred language of participants in this group. Interviews with the HREC group and Researcher group were primarily conducted in-person at participants' places of work, with one HREC group participant electing to answer the interview questions in written form and return by email.

\section{Sampling and participants}

Participants in the four groups were recruited through purposive sampling of the social and professional networks of the research team. For instance, all of the CALD group and most of the Family group were sourced through the Italian research assistant's (AB) networks in aged care and the broader Italian community. A shared cultural and linguistic identity facilitated an open and welcoming attitude towards the Italian research assistant by members of the Italian community, who either took part in the interviews personally or recommended suitable contacts in accordance with ethical approval for recruitment of participants. Focus group participants volunteered to take part during one of the research assistant's visits to a local Italian senior citizens' centre. The CALD group was provided with a verbal explanation of the project in Italian in accordance with ethics approval for this group. Their verbal consent was audio-recorded. The HREC group and Researcher group and some of the Family group were identified through the professional networks of other team members who work in medical and hospital environments in Melbourne. Depending on the method of initial contact, potential participants were invited to take part via email, or verbally in person with written documents provided. The Family, HREC and Researcher groups were provided with written information and a verbal explanation of the project and were asked to sign a consent form.

Table 1 summarises the profile of each group of informants and the purpose of including them in the study.

Ethics approval was obtained from The University of Melbourne (Project number 1443471).

\section{Data}

\section{Focus groups}

Although initially only interviews were planned, during data collection $\mathrm{AB}$ had an opportunity to interview two groups of older Italians in a community context. Focus groups are an effective and widely-used technique in health research as they provide a fertile environment from which to explore participants' knowledge, experiences and attitudes by capitalising on group processes and interactions to maximise data production. ${ }^{29}$ In the study, focus groups were appropriate for several reasons: firstly, they provided the chance to increase the number of participants, thereby increasing the variety of participants, e.g. in terms of language proficiency, education, experience in medical settings and use of interpretation services; secondly, open discussions in focus groups facilitate participant interactions, sharing of stories and comparing opinions; and lastly, vulnerable populations, such as CALD groups, may feel they have more safety in numbers. The topics discussed in the focus groups were identical to those discussed in CALD 
interviews.

\section{Interview questionnaires}

Four questionnaires were designed by the research team, and took into account medical, cultural, and linguistic factors potentially influencing CALD participation in medical research. The questionnaires for the CALD participants and their families sought to find out how participants (or their parents in the case of the Family group informants) interacted with the health system, their attitudes to research, and their use of and attitudes towards technology to determine the potential acceptability of a multimedia resource about medical research. The interview included questions regarding: age; language proficiency and use (of English, Italian and Italian dialect) in both an everyday and medical setting; education level; participants' familiarity with and use of technology; consent; and their experience with, and attitudes towards, medical research. The English version of the CALD group interview (Appendix 1) was pilot-tested with two of the researchers' parents, one of whom has a CALD background. The interview questionnaire for the HREC group included questions about the number and types of research projects reviewed by their committees, the proportion of patients whose primary language was not English, the protocols and provisions made for including these patients in research projects, informed consent procedures, and attitudes to supporting technology and the proposed tablet-based/iPad resource. The interview questionnaire for the Researcher group included questions about the research role of the interviewee, his/her level of experience, his/her estimate of the proportion of patients whose primary language was not English, protocols and provisions made for including these patients in research projects, informed consent procedures at their research hospital and his/her attitude to the proposed tablet-based resource.

\section{Analysis of interview data}

The interviews and focus groups were transcribed. Data relating to demographic features of the CALD and Family groups were extracted from the interviewer notes and transcriptions and tabulated in an Excel spread sheet. The remaining transcription data were then analysed thematically. First, open coding was performed: interview transcripts were read by $\mathrm{JHu}$ and $\mathrm{AB}$ line by line to identify ideas, events, or emotions, and to assign labels to these elements. Second, axial coding was performed to establish connections between major themes and sub-themes. The themes were independently examined by RWK to identify any overlap and redundancy. Differences were resolved by consensus.

\section{Results}

The findings are divided into two main sections. The first section reports the demographic characteristics of the CALD and Family groups and the thematic analysis of their interviews. The second section starts with the findings from the HREC participants' experiences in relation to ethics applications, including inclusion and exclusion criteria and consent processes in order to provide an overview of current practice. This overview is followed by the findings of the emergent themes from the HREC group and Researcher group.

\section{Linguistic, sociocultural profile of culturally and lin- guistically diverse and family group participants}

All of the CALD participants were born in Italy, none had completed high school and all except two had had only three to five years of schooling (in Italy before migrating). All Family group participants were daughters of older Italian Australians and were either born in Australia or arrived at an early age. All indicated that they helped their parent/s by attending medical appointments with them. Some helped all the time, including going to the local general practitioner (GP), while others assisted only for specialist appointments or hospital visits. At least half of the respondents had parents whose GP was Italian speaking.

Three main themes emerged from the thematic analysis of the data from the older Italians and family members. These themes were getting by in medical interactions; receptivity to medical research: testing the waters; and receptivity to technology for enabling understanding: passive versus active. Due to space constraints, we have included only one representative quote for each sub-theme. All CALD group quotes have been translated into English from Italian.

Table 1. Study participants.

\begin{tabular}{|c|c|c|}
\hline Participant group & Description of group & Purpose of interview \\
\hline CALD $(\mathrm{N}=21)$ & $\begin{array}{l}\text { Older individuals of Italian background. } \\
\text { Age range: } 59-90 \text {. Eight ( } 3 \text { male, } 5 \text { female) } \\
\text { were interviewed, individually or in pairs, } 13 \\
\text { ( } 7 \text { male, } 6 \text { female) participated in either of the } \\
\text { two focus groups. Interviews and focus groups } \\
\text { for CALD group took place between May and June } 2015\end{array}$ & $\begin{array}{l}\text { To find out about how participants interact with the health system, } \\
\text { attitudes to research; to determine acceptability of sample health } \\
\text { resource }\end{array}$ \\
\hline Family $(\mathrm{N}=10)$ & $\begin{array}{l}\text { Family members of older Italians (all daughters) } \\
\text { who are support persons of their relative in a } \\
\text { health/medical context. Interviews took place } \\
\text { in May and June } 2015\end{array}$ & $\begin{array}{l}\text { To find out how they assist their relative in interacting with the } \\
\text { health system; attitudes to research }\end{array}$ \\
\hline $\operatorname{HREC}(\mathrm{N}=4)$ & $\begin{array}{l}\text { Human Research Ethics Administrators } 4 \\
\text { ( } 2 \text { male, } 1 \text { female were interviewed individually, } \\
1 \text { female returned a written response to interview } \\
\text { questions by email). Interviews took place } \\
\text { in June and July } 2015\end{array}$ & $\begin{array}{l}\text { To find out how many researchers include CALD groups in their } \\
\text { research; attitudes to alternative consent processes; years of } \\
\text { involvement with ethics committee; number of projects submitted } \\
\text { for review each year }\end{array}$ \\
\hline
\end{tabular}

$\begin{array}{lll}\text { Researcher }(\mathrm{N}=4) & \text { Medical researchers from university- affiliated hospitals. } & \text { To find out how many researchers include CALD groups in their } \\ & \text { Four (all female) were interviewed individually. } & \text { research; experience of alternative consent processes; identify } \\ & \text { Interviews took place between June and August } 2015 & \text { key patient groups and their related access issues }\end{array}$

CALD, culturally and linguistically diverse; HREC, hospital human research ethics administrators. 


\section{Getting by in medical interactions}

The first theme for the CALD and Family groups refers to participants' reported tendencies to draw on their linguistic and family resources as well as hospital resources to manage the language demands of medical interactions. Sub-themes were: mixing languages, confusion, and anxiety. Linguistically, the CALD group participants reported that in their day-to-day lives they frequently spoke a mixture of everything: Italian, English and Italian dialect. The participants in the Family group similarly indicated mixing languages with their parents as they had varying levels of competence in Italian or dialect but were fluent in English. This behaviour carried over into medical appointments, for example:

Because you know, some medical things, I can't say it in Calabrese, cos I don't know that terminology. But she understands it if I say it in English.

(Family group)

Participants reported their uptake of supplementary language and comprehension support such as seeing Italian-speaking GPs and making use of interpreter services. Use of interpreting services, however, was limited, and participants gave conflicting reports of utility; some reported having excellent experiences while others questioned the accuracy. Family group informants were more resistant to the presence of interpreters:

They had an interpreter there and I'm thinking, No, I'm quite capable of interpreting for my mother.

(Family group)

Although CALD and Family group participants reported using their linguistic, family, and hospital-based resources, CALD group participants tended to assess their communicative competence as coping, and in several instances admitted to confusion. This assessment was echoed by family members:

Family Group participant:

And then I can explain it. Cos I just don't think Dad gets it all the time.

Interviewer:

Even when he's speaking to him [the GP/ in Italian?

Family Group participant:

Yeah. Dad gets embarrassed so he doesn't ask a lot of questions. Like, he'll just say "yes, yes, yes"

The sense of getting by was evidently problematic and insufficient when discussions touched on consent processes. The second subtheme, confusion, was apparent in the CALD and Family groups in relation to the meaning and purpose of consent, as can be seen from the following extract from one focus group discussion:

Interviewer:

Do you remember signing a consent [form]?

1: I think so.

2: Anything has to be signed.

3: They give you all the explanations, the paperwork to fill in. Interviewer: Paperwork in English?

Yes. And then my daughter comes. She would fill in the form.

4: Same for all of us.

A related sub-theme was anxiety: Family group participants also reported their parents feeling anxiety in consultations, which adversely affected their ability to comprehend.

See mum gets herself ... - anxiety kicks in. and when there's that anxiety, then the understanding (diminishes). ... She only half listens. And because she only half listened, she only half took it in, and then she's constantly asking me, 'But are you sure this, that and the other?'

(Family group)

In many cases, Family group participants had helped to explain procedures and consent forms to their parent, either in Italian or Italian dialect. All indicated that interpreters had never been present. Consent for surgery and for research participation was an area of concern for the Family group.

\section{Receptivity to medical research: testing the waters}

The second major theme for the CALD and Family groups was receptivity to medical research: testing the waters. The three interrelated sub-themes were willingness/ unwillingness, exclusion, and being useful. Most of the respondents in the CALD and Family groups demonstrated an understanding of medical research, and around half of both groups had been invited to participate in studies. There was variability in attitudes towards participating in medical research: a few participants from both groups had been involved in research studies previously, however only a few in each group indicated willingness to participate in medical research in the future. There was some indication that children of older Italians exerted a strong influence over their parents' decisions to participate in research:

Interviewer:

Have you participated in medical research?

CALD group (male, 83): Yes, more than once. Then another time I was invited to try a new medicine for diabetes, but my children discouraged me, so I refused.

Another sub-theme was inclusion and exclusion. Some CALD respondents believed that they would be excluded from participating because of low English proficiency:

I have never [been asked]. Especially when they understand you can't speak English.

(CALD group)

Others felt that researchers would not be interested in recruiting them:

I have too many health issues at the moment... Nobody wants to see me.

(CALD group)

Related to the sub-themes of willingness/unwillingness and exclusion was the sub-theme of being useful. One Family group participant suggested that a good way to encourage older people (of Southern European background) to get involved in research was by making them feel useful and needed:

If you tell an Italian they're needed, tell a Greek person that they're needed, you've got them. Seriously though, when it comes to things like that, they're not needed for anything anymore, old people. To be needed for something like that is HUGE.

(Family group) 
The children of older Italians also reported a cultural-generational willingness resulting from the awe in which their parents held doctors; that is, if a doctor told them to do something, they would do it:

But they're old school, if the doctors tell them to sign something, they'll do it. Without translating.

(Family group)

\section{Receptivity to technology for enabling understanding: passive versus active}

The third theme relates to participants' receptivity to enabling technology, and this is characterised as passive rather than active. In general, the CALD group did not use computers or modern technology such as smartphones or tablet computers, and were not familiar with the concept of 'apps', with the exception of one gentleman who was very keen to use any technology. In general, informants were aware of technology and reported their children using modern devices, but did not consider such technology relevant for them as users:

All these technologies have arrived too late for us. What should we do with them?

(CALD focus group)

I've seen it before but I've never used it.

(CALD focus group)

\section{Experiences and perceptions of hospital human research ethics committees and researcher group participants}

This second section of the results reports the findings for the HREC and Researcher groups. Four administrators from different hospital human research ethics committees (HREC Group) were interviewed, all of whom were CALD migrants to Australia from Europe or Asia. All participants commented on this aspect, noting their willingness to participate in research addressing barriers to CALD participation in medical research. The hospitals had varying research profiles, from a large teaching hospital that processed up to 60 high-risk projects per year and was involved in large multicentre trials, to a hospital that did not deal with high-risk projects but processed a moderate number of low-risk projects and quality assurance and audits (Table 2). In addition, four clinical researchers were interviewed (Researcher group). The respondents worked with all types of studies (high- and low-risk, small, multi-centre trials), quality assurance (QA) and audits. All researchers worked at different hospitals from each other, and the HREC administrators, aside from Researcher 2, who was as the same hospital as HREC 3.

\section{Consent processes}

The HREC and Researcher interviewees were asked to describe the types of consent that had been accepted by the ethics committees at their respective hospitals (Table 3 ). Written consent was the only

Table 2. Estimated number of research studies reviewed by hospital human research ethics administrators (HREC) at the HREC informants' hospitals per year.

\begin{tabular}{|c|c|c|c|c|}
\hline & HREC 1 & HREC 2 & HREC 3 & HREC 4 \\
\hline $\begin{array}{l}\text { Estimate of studies processed per year } \\
\text { High risk } \\
\text { Low risk } \\
\text { Quality assurance and audits }\end{array}$ & $\begin{array}{c}50-60 \\
\text { few } \\
40-50\end{array}$ & $\begin{array}{c}2 \\
200 \\
200\end{array}$ & $\begin{array}{c}0 \\
28 \\
121\end{array}$ & $\begin{array}{l}28 \\
61 \\
27\end{array}$ \\
\hline $\begin{array}{l}\text { Estimated n.\% trials involving CALD participants } \\
\text { (as primary or coincidental recruits) }\end{array}$ & $\begin{array}{l}1 \text { - primary } \\
(0.5 \%)\end{array}$ & $\begin{array}{c}5 \% \text { - primary; } \\
10 \% \text { - coincidental }\end{array}$ & $\begin{array}{c}1 \text { - primary; } \\
20 \text { - coincidental; } 7 \text { - excluded }\end{array}$ & $<10 \%$ \\
\hline
\end{tabular}

CALD, culturally and linguistically diverse.

Table 3. Types of consent accepted in respective hospitals according to hospital human research ethics administrators (HREC) and researcher informants.

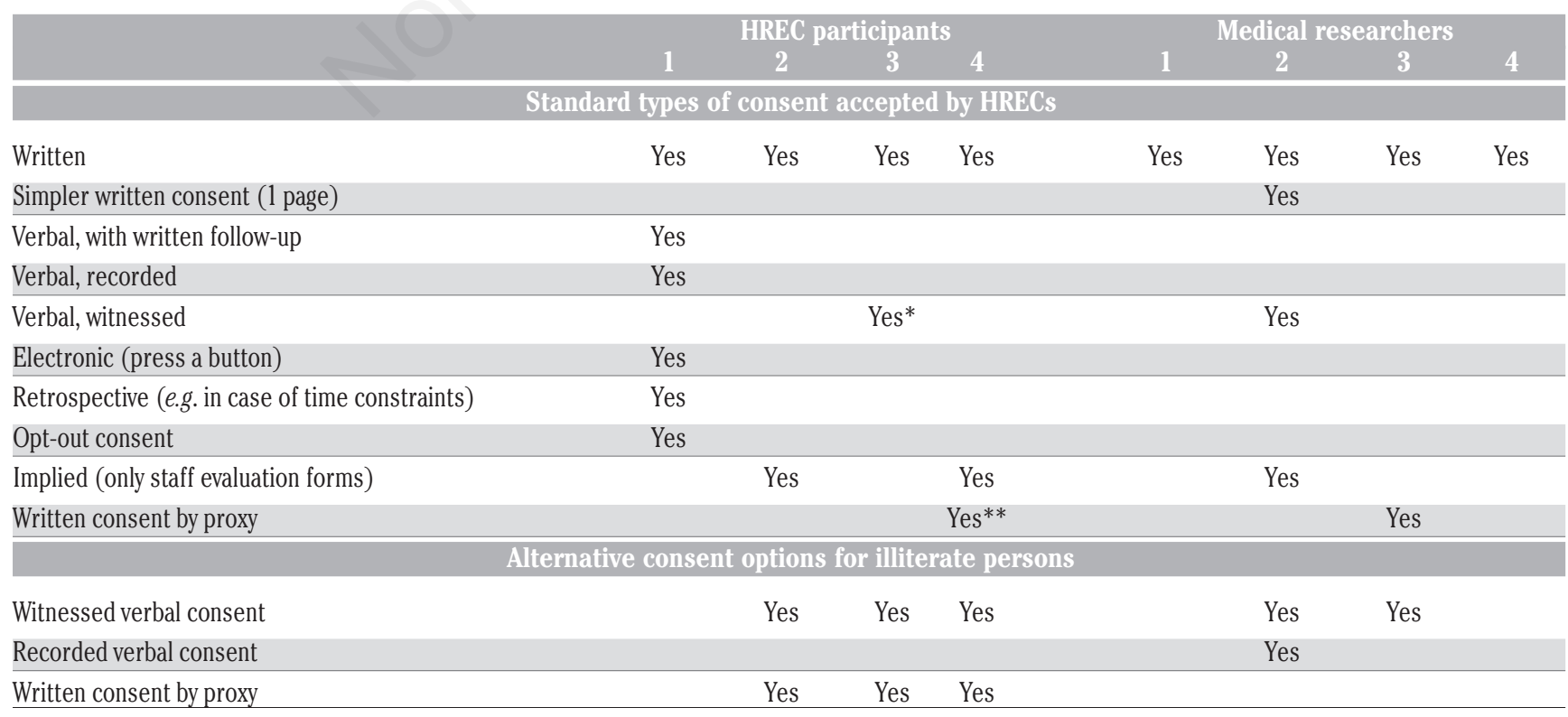

*Rarely accepted. **Proxies are parents whose children are undergoing treatment, and adult children with cognitively impaired elderly parents. 
form of consent accepted in most cases except for staff evaluations where the act of completion implies consent. In several cases, no alternative (i.e. non-written) modes of consent were available for people who are illiterate (in English and potentially their own language also). Also, while HREC 3 and Researcher 2 were from the same institution, their responses were somewhat different, with Researcher 2 noting a wider range of consent mechanisms than HREC 3 (apart from written consent by proxy).

\section{Findings of thematic analysis: experiences of hospital human research ethics committees administrators, and researchers}

Three major themes with sub-themes about CALD patient participation in research emerged from the interview data from these two groups: exclusion, incorporating sub-themes language and literacy barriers, and resource barriers; cultural factors, with sub-themes mistrust and shared decision making; and e-consent, with the sub-themes of acceptability, and of education and initiatives.

\section{Exclusion}

This theme refers to the exclusion of CALD participants as evident in ethics applications and in recruitment for trials. HREC group participants reported that very few ethics applications to their HREC catered for non-English speaking participants, and all except one had difficulty remembering any specific projects. Researcher informants affirmed that the vast majority of their projects excluded non-English speakers. Two related sub-themes were language and literacy barriers, and resourcing to overcome these barriers. Respondents commented that while translation services and interpreters were present in the hospitals, these were primarily for patient care. Researchers reported being generally therefore restricted in their ability to work with interpreters for research purposes.

For studies where we don't have the resources to provide translation and I think that it's required, then we would specifically exclude participants whose comprehension wasn't adequate to be able to sign their consent form. (Researcher)

Other factors concerning language and literacy issues related to needing to book interpreters ahead of time, which may not be feasible in the recruitment context, and language difficulties with follow-ups, which are usually in the form of phone calls.

\section{Cultural factors}

The second major theme to emerge from the HREC and Researcher groups was the effect of cultural differences on recruiting CALD participants. Mistrust of institutions and research was one of the sub-themes. Mistrust leads people with particular backgrounds to decline to participate in research, but it also discouraged researchers from seeking to enrol members of these groups because they were aware that it might be a negative or threatening experience for them.

I think also one of the struggles that people do mention is that a lot of the patients that we get automatically think that research is a bad thing, probably because of where they have come from. So people are reluctant to approach them for that reason as well. (HREC group)

A second sub-theme was the central role that family can play in decision-making about participation in research, a factor that was deemed to be more prevalent in CALD background families.
Interviewer:

So family dynamics play a big part in whether they get involved or not?

Researcher: I think definitely. Especially the ones where English is their second language. It's really very much about family and family consent.

\section{Electronic (E-)consent}

The third major theme, e-consent, refers to participant conjectures about the likelihood of an electronic form of consent such as an audio-visual or an online interactive form being acceptable to ethics review boards, and to trial researchers and recruiters. The acceptability of a proposed multimedia resource to explain a clinical trial and which included an electronic form of consent incorporating CALD languages and multimedia interfaces was positive amongst these informants. There was a perception that it could be a positive move forward in general, not only with CALD populations. Participants felt that people would be able to understand and retain more information because of the multimedia audio-visual approach, and that it would ensure that information provision was given in the same way to all.

As time goes on and everything is becoming more digital and technology-based, I think this is probably a better way to go. ... And I think it will eliminate certain groups from being excluded from studies. Like people who are illiterate. They can still hear and understand and they'll be able to be a participant. Because I think that in a way you are biasing studies by not including certain groups of people. You're just targeting a certain group even though you should be including everyone. So that would help to overcome that barrier as well. (Researcher)

However, many respondents expressed reticence about HRECs' acceptance of such an innovation, and anticipated they would have strong concerns and requirements regarding any proposed electronic consent processes.

I think it's fantastic [ a proposed resource incorporating video and video consent]. Because I think you can gain more from seeing somebody understand - from a video. And they will pick up more from having everything explained to them, what the research is about and everything. I can see a lot of benefit from it personally. I think the older people on the committee might be the ones who object more. (HREC participant)

I think getting the diverse range of ethics committees to agree to non-conventional modes of consent would be the biggest sticking point. (Researcher)

In addition to the sub-theme of acceptability, participants underscored the need for education of ethics committees and educational initiatives to enable innovations in consent processes to gain traction and acceptability. The general perception was that a process of education for HRECs would be needed before they would consider a novel format such as a multimedia tablet-based resource for non-English speakers with electronic participation information and interactive consent.

There is still a slow uptake of ethics committees accepting this change and it would probably require education and clarification of privacy and how consent is verified via e-consent. (HREC participant)

Nonetheless, there was acknowledgment from the HREC and 
Researcher groups that HRECs were aware of the need to modify practices and investigate ways to help overcome the lack of inclusion of CALD groups in clinical research. One HREC administrator stated that her committee was thinking about ways to overcome barriers to CALD participation in clinical research, including collaborating with the language services department in the planning stages of research projects, or bringing a language services representative onto the committee. Another respondent's committee was considering ways to simplify long and complex consent documentation in order to make it accessible to CALD people with low levels of literacy, for instance by providing a translated summary version.

\section{Discussion and implications}

The aims of this qualitative study were to investigate the barriers to CALD patients participating in medical research from the perspectives of four stakeholder groups: older Italian-Australians, their family members, HREC administrators, and researchers. The study also explored the potential of a tablet-based resource to overcome these barriers and increase trial participation of CALD groups.

Our findings confirm that there were considerable cultural, linguistic, and resourcing barriers hindering and excluding the participation of older Italian-Australians in medical research, findings which resonate broadly with existing research on barriers to people of CALD backgrounds' participation in clinical trials, and which here have been investigated from the four stakeholder perspectives. Furthermore, our findings showed that in this study setting there were few enabling strategies currently in place to address these barriers despite the national ethics guidelines for equitable participation in research.

The themes for the CALD group and the family group were aligned and were therefore reported together; the first of these namely, getting by in medical interactions with sub- themes of mixing languages, confusion, and anxiety referred both to medical interactions as well as experiences of trial participation and recruitment. This theme and the receptivity sub-theme of exclusion, resonated with the major theme of exclusion for the HREC and researcher groups, which identified tangible aspects such as funding for interpreters, and patients' language and understanding that hindered recruitment of older patients from CALD backgrounds. The second theme of the HREC group and researcher group, cultural factors with sub-themes mistrust and shared decision-making, similarly resonated with Italian background group sub-themes of willingness/unwillingness, and anxiety. Furthermore, ethics practices may play a significant role in contributing to low participation rates. Researchers and HREC administrators mentioned two tangible issues limiting CALD participants taking part in medical research in the Australian setting: ethical concerns about the ability to ensure adequate communication and understanding between CALD patients and researchers, and a lack of available research or hospital funding for language services (translating and interpreting) to address the communication barriers. This finding is consistent with the existing international literature. ${ }^{30,31}$ One noticeable difference was the Italian background informants' (CALD and family groups') identification of factors that could be used as enablers for participation, an aspect that did not feature in HREC and researcher responses. Previous research has shown that people are motivated to participate in research, for various personal and group/community-related reasons. ${ }^{32}$ The willingness to engage in research reported by some CALD patients and their children in our data centred around being useful or needed, as well as their respect for clinicians as opposed to their distrust of medical research. These factors should be taken into account when designing educative tools and patient information about medical research for this patient group. Furthermore, the reported elderly participants' linguistic strategy of mixing languages could be taken into account as an enabler when translating and interpreting participant information; it could also assist family discussions in the reported process of the family dimension of decision making.

Data from our interviews support the view that education of the patient and family groups and engagement with CALD communities are important steps in addressing the low participation of CALD groups in research. ${ }^{33}$ Among the patient group, education is needed to address mistrust of institutions and research, a commonly cited barrier to research recruitment in minority and CALD groups. ${ }^{34-36}$ This could be achieved through initiatives such as the Let's Talk Medical Research resource (described below), which aims to explain the principles and purpose of clinical research in simple terms, in the preferred language of the listener. It is also important to include families in such education initiatives, given that families of CALD patients may be more involved in decision-making processes than those of nonCALD patients, and act as gatekeepers in many instances. Connected to this last point is the reported resistance to the presence of interpreters by some family members, a new finding to emerge from this study, and one that may have bearing on recruitment efforts and research design, especially given the noted importance of shared decision-making. Our findings that education and consultation may be needed for HREC committee members in order to consider novel but culturally and linguistically appropriate forms of consent align with existing findings for recruitment of culturally and linguistically diverse groups. ${ }^{37}$ This is needed as in many research settings the shortage of research funding is a major constraint in providing translations and interpreters for recruitment. More research on alternative forms of consent in CALD populations and communication about the outcomes with ethics committees is one way to help this process.

The findings of the current study are limited by the small sample size for all groups. For example, a larger sample size of researchers may identify additional barriers. It is also noteworthy that all of the Family group participants were females. However, since this feature fits with the general profile of the family carer, we consider that an allfemale sample for this group is fairly representative.

\section{Future directions and outcomes: an educational tool about medical research}

The findings of the study, as well as the findings of a literature review on the efficacy of multimedia tools for medical research and education purposes, ${ }^{24}$ informed the design of a prototype multimedia resource. The tablet format of the resource makes it versatile and portable, suitable for use by trial administrators, health professionals, migrant health services with CALD patients and their families, and in community settings. The resource called Let's talk medical research: parliamo di ricerca medica is bilingual with navigation in English and Italian, includes video supported by written key information (and optional audio) about the purpose and process of medical research, and ethical considerations. The narrator is a medical doctor. Other features are a vignette from a highly regarded researcher of Italian background, a brief re-enactment of a patient being asked about participation in a clinical trial, as well as audio-recording functionality to test participants' ability to use the resource with guidance. The resource will be trialled at a metropolitan hospital in 2016. The findings of the prototype trial will be disseminated to ethics committees for discussion about receptivity to electronic forms of consent for this patient group.

Further research is needed to explore the extent to which family members influence patients' decision to participate in trials as well as other socio-economic factors and health literacy. 


\section{Conclusions}

People from CALD backgrounds in migrant destination countries such as Australia remain under-represented in clinical trials, a factor that can contribute to ongoing health inequalities for these groups as well as compromising the generalisability of trial outcomes. This innovative study engaged four stakeholder groups: older Italians, their families, ethics committee administrators, and medical researchers, to examine the barriers and enablers to participation, including the acceptability of multimedia resources to provide education and a consent mechanism to research participation. The findings have informed the development of a resource that is tailored to the cultural and linguistic needs of an ageing and established migrant group, and also holds the potential to be adapted for use with other CALD groups.

Correspondence: Anna Parker, Melbourne Medical School, University of Melbourne, Parkville, VIC 3010, Australia.

Tel.: +61.3.9035.9662.

E-mail: anna.parker@unimelb.edu.au

Key words: Culturally and linguistically diverse; medical research; informed consent; multimedia; migrant health.

Acknowledgments: the authors would thank Melbourne Networked Society Institute (MNSI), University of Melbourne, for a 2014 project grant; the ItaloAustralian participants and their families; the research ethics administrators, trial coordinators, and the anaesthesia researchers who participated in the study; from MNSI, Dr Chamil Jayasundara, and Mr Ken Clarke for resource development; and Dr Sean Fabri, Professor Rinaldo Bellomo, and Ms Maria Phieler for appearing on the resource; and Mrs Mardi Story and Mrs Britt Wilson for pilot testing the interviews.

Contributions: RWK, JHu, JHa, AP, AB contributed to the conception and design, acquisition, analysis and interpretation of data; the drafting of the article and/or revising it for important intellectual content; and final approval of the version to be published; DS and UK contributed to the conception and design; the revising of the article for important intellectual content; and final approval of the version to be published; TDP was responsible for the initial conception of the project; revising of the article for important content and gave final approval of the version to be published.

Conflict of interest: the authors declare no potential conflict of interest.

Received for publication:10 December 2015.

Accepted for publication: 27 January 2016.

CCopyright R. Woodward-Kron et al., 2016

Licensee PAGEPress, Italy

Journal of Public Health Research 2016;5:667

doi:10.4081/jphr.2016.667

This work is licensed under a Creative Commons Attribution NonCommercial 4.0 License (CC BY-NC 4.0).

\section{References}

1. Greenstock L, Woodward-Kron R, Fraser C, et al. Telecommunications as a means to access health information: an exploratory study of migrants in Australia. J Public Health Res 2012;1:216-21.

2. Henderson S, Kendall E, See L. The effectiveness of culturally appropriate interventions to manage or prevent chronic disease in culturally and linguistically diverse communities: a systematic lit- erature review. Health Soc Care Community 2011;19:225-49.

3. Ethnic Communities Council of Victoria. An investment not an expense: enhancing health literacy in CALD communities. Ethnic Communities Council of Victoria, 2012. Available from: http://fecca.org.au/wp- content/uploads/2015/06/national-cald-agedcare-strategy.pdf. Accessed on: 2 July 2015.

4. Hernández MG, Nguyen J, Casanova S, et al. Doing no harm and getting it right: guidelines for ethical research with immigrant communities. New Dir Child Adolesc Dev 2013;2013:43-60.

5. Jones C, Jablonski RA. I don't want to be a Guinea pig: recruiting older African Americans. J Gerontol Nurs 2014;40:3-4.

6. Liu JJ, Davidson E, Sheikh PA. Achieving ethnic diversity in trial recruitment. Pharm Med 2012;25:215-22.

7. National Health and Medical Research Council. Potential benefits and risks Australian Clinical Trials. 2015. Available from: http://www.australianclinicaltrials.gov.au/why-be-part-clinicaltrial/potential-benefits- and-potential-risks. Accessed on: 7 December 2015.

8. Australian Bureau of Statistics. census community profiles: Brimbank. 2011. Available from: http://www.censusdata.abs.gov .au/census_services/getproduct/census/2011/communityprofile/21 301?opendocument\&navpos=220. Accessed on: 23 November 2015.

9. Giuliano AR, Mokuau N, Hughes C, et al. Participation of minorities in cancer research: the influence of structural, cultural, and linguistic factors. Ann Epidemiol 2000;10:S22-34.

10. National Health and Medical Research Council. National statement on ethical conduct in human research. 2007. Available from: https://www.nhmrc.gov.au/_files_nhmrc/publications/attachments/e72_national_statemen t_may_2015_150514_a.pdf

11. Glickman SW, Ndubuizu A, Weinfurt KP, et al. Perspective: the case for research justice: inclusion of patients with limited English proficiency in clinical research. Acad Med J Assoc Am Med Coll 2011;86:389-93.

12. Tait AR, Voepel-Lewis T. Digital multimedia: a new approach for informed consent? JAMA 2015;313:463-4.

13. Gostin LO. Informed consent, cultural sensitivity, and respect for persons. JAMA 1995;274:844-5.

14. Bhutta ZA. Beyond informed consent. Bull World Health Organ 2004;82:771-7.

15. Stead M, Eadie D, Gordon D, Angus K. Hello, hello - it's English I speak!: a qualitative exploration of patients' understanding of the science of clinical trials. J Med Ethics 2005;31:664-9.

16. Walker C, Weeks A, McAvoy B, Demetriou E. Exploring the role of self-management programmes in caring for people from culturally and linguistically diverse backgrounds in Melbourne, Australia. Health Expect 2005;8:315-23.

17. Killawi A, Khidir A, Elnashar M, et al. Procedures of recruiting, obtaining informed consent, and compensating research participants in Qatar: findings from a qualitative investigation. BMC Med Ethics 2014;15:9.

18. Henderson S, Kendall E. Culturally and linguistically diverse peoples' knowledge of accessibility and utilisation of health services: exploring the need for improvement in health service delivery. Aust J Prim Health 2011;17:195-201.

19. Nehme J, El-Khani U, Chow A, et al. The use of multimedia consent programs for surgical procedures: a systematic review. Surg Innov 2013:20:13-23.

20. Sonne SC, Andrews JO, Gentilin SM, et al. Development and pilot testing of a video-assisted informed consent process. Contemp Clin Trials 2013:36:25-31.

21. Farrell EH, Whistance RN, Phillips K, et al., Systematic review and meta-analysis of audio-visual information aids for informed consent for invasive healthcare procedures in clinical practice. Patient Educ Couns 2014:94:20-32. 
22. Hughson J, Woodward-Kron R, Parker A, et al. A review of approaches to improve participation of culturally and linguistically diverse populations in clinical trials. Trials. [In Press].

23. Santoyo-Olsson J, Cabrera J, Freyre R, et al. An innovative multiphased strategy to recruit underserved adults into a randomized trial of a community-based diabetes risk reduction program. Gerontologist 2011;51:S82-93.

24. Kauffman KS, dos Reis S, Ross M, et al. Engaging hard-to-reach patients in patient-centred outcomes research. J Comp Eff Res 2013;2:313-24.

25. Ejiogu N, Norbeck JH, Mason MA, et al. Recruitment and retention strategies for minority or poor clinical research participants: lessons from the healthy aging in neighborhoods of diversity across the life span study. Gerontologist 2011;51:S33-45.

26. Australian Bureau of Statistics. Census community profiles: greater Melbourne. 2011 Available from: http://www. censusdata.abs.gov.au/census_services/getproduct/census/2011/co mmunityprofile/2GMEL?opendocument\&navpos=220. Accessed on: 22 October 2015.

27. Australian Bureau of Statistics. Main Features. Where do migrants live? Available from: http://www.abs.gov.au/ausstats/abs @.nsf/Lookup/4102.0main+features102014\#MELBOURNE. Accessed on: 23 November 2015.

28. Australian Bureau of Statistics. 2011 census data. Australian Government; 2015.
29. Kitzinger J. Qualitative research: introducing focus groups. BMJ 1995;311:299-302.

30. Lloyd CE, Johnson MR, Mughal S, et al. Securing recruitment and obtaining informed consent in minority ethnic groups in the UK. BMC Health Serv Res 2008;8:68.

31. Howerton MW, Gibbons MC, Baffi CR, et al. Provider roles in the recruitment of underrepresented populations to cancer clinical trials. Cancer 2007;109:465-76.

32. Clark T. On being researched: why do people engage with qualitative research? Qual Res 2010;10:399-419.

33. Lwin Z, Broom A, Cosman R, et al. Culturally and linguistically diverse patient participation in glioma research. Neuro-Oncol Pract 2014;1:101-5.

34. Barata PC, Gucciardi E, Ahmad F, Stewart DE. Cross-cultural perspectives on research participation and informed consent. Soc Sci Med 2006;62:479-90.

35. Moreno-John G, Gachie A, Fleming CM, et al. Ethnic minority older adults participating in clinical research: developing trust. J Aging Health 2004;16:93S-123S.

36. Pirie A, Gute DM. Crossing the chasm of mistrust: collaborating with immigrant populations through community organizations and academic partners. Am J Public Health 2013;103:2126-30.

37. Marshall PA. Cultural competence and informed consent in international health research. Camb Q Healthc Ethics 2008;17:206-15. 